\title{
Activation of ARK5/miR-1181/HOXA10 axis promotes epithelial-mesenchymal transition in ovarian cancer
}

\author{
HAI-YAN ZHANG ${ }^{1}$, JIAN-HUA LI ${ }^{2},{\text { GUANG } \mathrm{LI}^{3} \text { and SU-RONG WANG }}^{4}$ \\ ${ }^{1}$ Gynaecology Ward-1, Linyi City People's Hospital; ${ }^{2}$ Lab, Linyi City People's Hospital; ${ }^{3}$ Gastrointestinal Surgery, \\ Linyi City People's Hospital; ${ }^{4}$ Gynaecology Ward-3, Linyi City People's Hospital, Shandong 276003, P.R. China
}

Received January 25, 2015; Accepted April 3, 2015

DOI: 10.3892/or.2015.4113

\begin{abstract}
Epithelial ovarian cancer (EOC) is the sixth most common cancer in females worldwide and, although advances have been made in the detection, diagnosis and therapies for EOC, it remains the most lethal gynecologic malignancy in advanced countries. Nevertheless, relatively little is known concerning the molecular events that lead to the development of this highly aggressive disease. Elucidating the molecular mechanism involved in this disease may prove useful to understand the pathogenesis and progression of the disease, and to identify new targets for effective therapies. In the present study, we examined the role of ARK5 in ovarian cancer and normal matched tissues using western blot analysis and migration and invasion, and wound-healing assays. The results showed that ARK5 was upregulated in ovarian cancer tissues, compared with adjacent normal tissues. Moreover, it promoted epithelial-mesenchymal transition (EMT) and inhibited miR-1181 expression in ovarian cancer cells. Subsequent investigations showed that miR-1181 promoted mesenchymal-epithelial transition (MET) in ovarian cancer cells. Downstream target genes of miR-1181 were searched, and it was identified that miR-1181 degraded HOXA10 by targeting its 3' untranslated region (3'UTR) in ovarian cancer cells. The results confirmed that HOXA10 promoted EMT in ovarian cancer cells. Thus, activation of the ARK5/miR-1181/HOXA10 axis may be positively associated with EMT in ovarian cancer.
\end{abstract}

\section{Introduction}

Epithelial-mesenchymal transition (EMT) is an essential process for driving plasticity during development, and an

Correspondence to: Dr Jian-Hua Li, Lab, Linyi City People's Hospital, Shandong 276003, P.R. China

E-mail: lijianhua2015@yahoo.com

Abbreviations: EMT, epithelial-mesenchymal transition; MET, mesenchymal-epithelial transition

Key words: ARK5, miR-1181, HOXA10, ovarian cancer unintentional behavior of cells during the progression of malignant tumor (1-3). The EMT-associated reprogramming of cancer cells suggests that fundamental changes occur to several regulatory networks and that an intimate interplay exists between them. Disturbance of a controlled epithelial balance is triggered by altering several layers of regulation, including the transcriptional and translational machinery, expression of non-coding RNAs, alternative splicing and protein stability (4-6).

ARK5, known as KIAA0537/ Novel (nua) kinase family 1 (NUAK1) has been identified as the fifth member of the adenosine monophosphate-activated protein kinase (AMPK)related kinase (ARK) family (7). Akt phosphorylates ARK5 at Ser600, a C-terminal site outside the catalytic domain, which leads to activation of the 74-kDa kinase. During glucose deprivation or response to adenosine monophosphate, ARK5 supports cell survival in an Akt-dependent manner (7). It suppresses cell death induced by nutrient starvation and activation of death receptors through inhibition of caspase 8 , and the negative regulation of procaspase $6(8,9)$. ARK5 is also strongly associated with tumor invasion and metastasis, and is a tumor survival and tumor progression factor (10-18).

MicroRNAs (miRNAs) are small non-coding RNA molecules that suppress gene expression by interacting with the 3' untranslated regions (3'UTRs) of target mRNAs (19-21). The aberrant expression of miRNAs are reported in various human types of cancer and are known to have an oncogenic or tumor-suppressor role and have been shown to play key roles in cell survival, proliferation, apoptosis, migration, invasion and various other characteristic features that are altered in human cancer types $(22,23)$. Over $50 \%$ of the known miRNAs have been shown to participate in human tumorigenesis and/or metastasis by directly targeting oncogenes or tumor-suppressor genes $(24,25)$. miR-1181 inhibits stem cell-like phenotypes and suppresses SOX2 and STAT3 in human pancreatic cancer, although its role has not been reported in ovarian cancer (26). In addition, EMT is associated with acquisition of malignant and stem-cell characteristics (27).

In the present study, we examined the role of ARK5 in in ovarian cancer tissues, compared with normal matched tissues. The results showed that ARK5 was upregulated in the cancer tissues versus the healthy tissues. Moreover, ARK5 promoted EMT and inhibited miR-1181 expression in ovarian cancer cells. Subsequent investigations showed that miR-1181 
promoted MET in ovarian cancer cells. We also searched downstream target genes of miR-1181, and found that miR-1181 degraded HOXA10 by targeting its 3'UTR in ovarian cancer cells. Additionally, we confirmed that HOXA10 promoted EMT in ovarian cancer cells. Thus, activation of the ARK5/ miR-1181/HOXA10 axis may be positively associated with EMT in ovarian cancer.

\section{Materials and methods}

Ovarian cancer tissues. Six ovarian cancer patients diagnosed with ovarian cancer were recruited from the Department of Obstetrics and Gynaecology, Linyi People's Hospital and Hubei Cancer Center. Human tissue samples were utilized according to internationally recognized guidelines as well as local and national regulations. Studies carried out on human subjects were conducted according to international and national regulations. The Medical Ethics Committee approved the experiments undertaken. Informed consent was obtained from each participant.

Cell lines, plasmids, pre-miR-1181/control miR, antimiR-1181/scramble and transfection. Human EG, OVCAR8, OVCAR3, OCC1, HEY and SKOV3 ovarian cancer cell lines were obtained from the MD Anderson Cancer Center (Houston, TX, USA). Briefly, the cells were maintained in RPMI-1640 medium supplemented with 5\% fetal bovine serum (FBS) (Gibco, Grand Island, NY, USA) and penicillin/streptomycin at $37^{\circ} \mathrm{C}$ in a humidified atmosphere with $5 \% \mathrm{CO}_{2}$. ARK5- and HOXA10-expressing plasmids/empty vectors (pcDNA3.1) were purchased from the National RNAi Core Facility in Academic Sinica, Taipei, Taiwan. The expressing plasmids or empty vector (pcDNA3.1) used for each transfection was $10 \mu \mathrm{g}$. Pre-miR-1181/control miR and anti-miR-1181/ scramble were purchased from Ambion, Inc. (Austin, TX, USA). Transfection was performed with Lipofectamine 2000 reagent (Invitrogen, Carlsbad, CA, USA) according to the manufacturer's instructions.

Western blot analysis. Western blot analysis was performed as previously described (28). Briefly, after incubation with primary antibody anti-ARK5 (1:500), anti-E-cadherin (1:500), anti- $\alpha$-catenin (1:500), anti-N-cadherin (1:500), anti-vimentin (1:500), anti- $\alpha$-SMA (1:500), anti- $\beta$-catenin (1:500), anti-fibronectin (1:500), anti-SNAI1 (1:500), anti-TWIST (1:500), anti-TGFB1 (1:500), anti-ZEB1 (1:500), anti-TGFB2 (1:500), anti-HOXA10 (1:500) and anti- $\beta$-actin (1:500) (all from Abcam, Cambridge, MA, USA) overnight at $4^{\circ} \mathrm{C}$, IRDye ${ }^{\mathrm{TM}} 800$-conjugated anti-rabbit secondary antibodies (LI-COR Biosciences, Lincoln, NE, USA) were used for $30 \mathrm{~min}$ at room temperature. The specific proteins were visualized by the Odyssey ${ }^{\mathrm{TM}}$ Infrared Imaging System (Gene Company, Lincoln, NE, USA).

Migration and invasion assays. For the Transwell migration assays, $3 \times 10^{4}$ cells were plated in the top chamber with the non-coated membrane (24-well insert; pore size, 8-mm; BD Biosciences, San Jose, CA, USA). For the invasion assays, $1.25 \times 10^{5}$ cells were plated in the top chamber with Matrigel-coated membrane (24-well insert; pore size, $8 \mathrm{~mm}$; BD Biosciences). In the two assays, the cells were plated in medium without serum or growth factors, and medium supplemented with serum was used as a chemoattractant in the lower chamber. The cells were incubated for $36 \mathrm{~h}$ and any cells that did not migrate or invade through the pores were removed using a cotton swab. Cells on the lower surface of the membrane were stained with the Diff-Quik Staining Set (Dade Behring, Newark, DE, USA) and counted.

Wound-healing assay. Cells $\left(5 \times 10^{5}\right)$ were seeded in each 35-mm glass bottom dish (MatTek Corporation, Ashland, MA, USA) and cultured at $37^{\circ} \mathrm{C}$ with $5 \% \mathrm{CO}_{2}$ for $24 \mathrm{~h}$. The confluent monolayer of cells was wounded. Cell monolayers were wounded with yellow pipette tips. After washing with warm phosphate-buffered saline (PBS), the cells were incubated in fresh culture medium. The wounded areas were photographed at the beginning $(0 \mathrm{~h}$, top panels) and the end $(10 \mathrm{~h}$, bottom panels) of the assay using a Nikon inverted microscope (Eclipse TE-2000U) equipped with a video camera (DS-U1) (both from Nikon, Japan).

Immunocytochemistry. Cells transfected as indicated and grown in chamber slides (Laboratory-Tek; Nalge Nunc International, Rochester, NY, USA) were washed 3-4 times with PBS and fixed with $4 \%$ formaldehyde for $15 \mathrm{~min}$ at room temperature. After 3-4 PBS washes, the cells were permeabilized with $0.25 \%$ Triton X-100 in PBS. The cells were incubated in PBS containing $2 \%$ BSA, followed by overnight incubation at $4^{\circ} \mathrm{C}$ with anti-E-cadherin or anti-vimentin antibodies. The secondary antibody was incubated for $2 \mathrm{~h}$ at room temperature.

miRNA microarray. Total RNA from cultured cells, with efficient recovery of small RNAs, was isolated using the mirVana miRNA Isolation kit (Ambion, Inc.). cRNA for each sample was synthesized using a 3' IVT Express kit (Affymetrix, Santa Clara, CA, USA) according to the manufacturer's instructions. The purified cRNA was fragmented by incubation in fragmentation buffer (provided in the 3'IVT Express kit) at $95^{\circ} \mathrm{C}$ for $35 \mathrm{~min}$ and chilled on ice. The fragmented labeled cRNA was applied to MicroRNA2.0 array and hybridized in a GeneChip Hybridization Oven 640 (both from Affymetrix) at $45^{\circ} \mathrm{C}$ for $20 \mathrm{~h}$. After washing and staining in a GeneChip fluidics station 450, the arrays were scanned using a GeneChip Scanner 3000 (both from Affymetrix). The gene expression levels of samples were normalized and compared using Partek GS 6.5 (Partek Inc., St. Louis, MO, USA). Average-linkage hierarchical clustering of the data was applied using the cluster and the results were shown using TreeView (both from Stanford, Stanford University, CA, USA; http://rana.lbl.gov).

Reverse transcription-qPCR for miRNA. Total RNA from cultured cells, with efficient recovery of small RNAs, was isolated using the mirVana miRNA Isolation kit. Detection of the mature form of miRNAs was performed using the mirVana RT-qPCR miRNA Detection kit, according to the manufacturer's instructions (Ambion, Inc.). The U6 small nuclear RNA was used as an internal control.

Bioinformatics analysis. An analysis of potential miRNA target sites was performed using prediction algorithms by miRanda (http://www.microrna.org/microrna/home.do). 
Immunofluorescence analysis. For the immunofluorescence analysis, the cells were plated on glass coverslips in 6-well plates and transfected as indicated. At $48 \mathrm{~h}$ after transfection, the coverslips were stained with the abovementioned antiHOXA10 antibodies. Alexa Fluor 488 goat anti-rabbit IgG antibody was used as the secondary antibody (Invitrogen). The coverslips were counterstained with DAPI (Invitrogen Molecular Probes, Eugene, Oregon, USA) for visualization of the nuclei. Microscopic analysis was performed with a confocal laser-scanning microscope (Leica Microsystems, Bensheim, Germany). Fluorescence intensities were measured in a few viewing areas for 200-300 cells/coverslip and analyzed using ImageJ $1.37 \mathrm{v}$ software (http://rsb.info.nih.gov/ij/index.html).

Reverse-transcription polymerase chain reaction (RT-PCR) and RT-qPCR for HOXA10. Total RNA was isolated from cells using TRIzol reagent (Invitrogen). First-strand cDNA was synthesized from the total RNA using M-MLV reverse transcriptase (Promega, Madison, WI, USA) and random hexamer primers (Sangon, Shanghai, China). The thermal cycle profile used was: denaturation for $30 \mathrm{sec}$ at $95^{\circ} \mathrm{C}$, annealing for $45 \mathrm{sec}$ at $53-58^{\circ} \mathrm{C}$ depending on the primers used, and extension for $45 \mathrm{sec}$ at $72^{\circ} \mathrm{C}$. PCR products were visualized on $2 \%$ agarose gels stained with ethidium bromide under UV transillumination. RT-qPCR was carried out with a Power SYBR-Green PCR Master Mix (Applied Biosystems, Carlsbad, CA, USA) according to the manufacturer's instructions. The primer sequences used for HOXA10 were: forward, 5'-GCCCTTCCGAGAGCAGCAAAG-3' and reverse, 5'-AGG TGGACGCTGCGGCTAATCTCTA-3'.

Luciferase reporter assay. The 3'-UTR of human HOXA10 mRNA was cloned in pRL-TK (Promega) using a PCR-generated fragment. Site-directed mutagenesis of the miR-1181 target-site in the HOXA10-3'-UTR was carried out using a QuikChange mutagenesis kit (Stratagene, Heidelberg, Germany), with HOXA10-WT-luc as a template. For reporter assays, cells was transiently transfected with WT or mutant reporter plasmids and miRNA or anti-miRNA (as shown in Fig. 5H and I) using Lipofectamine 2000 (Invitrogen). Reporter assays were performed $36 \mathrm{~h}$ post-transfection using the DualLuciferase Reporter Assay System (Promega), normalized for transfection efficiency by co-transfected Renilla luciferase.

\section{Results}

ARK5 is upregulated in ovarian cancer tissues. To identify ARK5 protein expression in ovarian cancer tissues, we performed western blot analysis to detect ARK5 protein between ovarian cancer and adjacent normal tissues. We found that ARK5 was increased in the cancer tissues of 6 patients, compared with the adjacent normal tissues (Fig. 1). The results suggested that ARK5 may be an oncogene in ovarian cancer.

ARK5 promotes EMT in ovarian cancer cells. Western blotting was performed to identify the ARK5 protein expression in the EG, OVCAR8, OVCAR3, OCC1, HEY and SKOV3 ovarian cancer cell lines. Protein isolated from the six cell lines was detected by western blotting and the results showed that the expression of ARK5 was lowest in EG cells, whereas

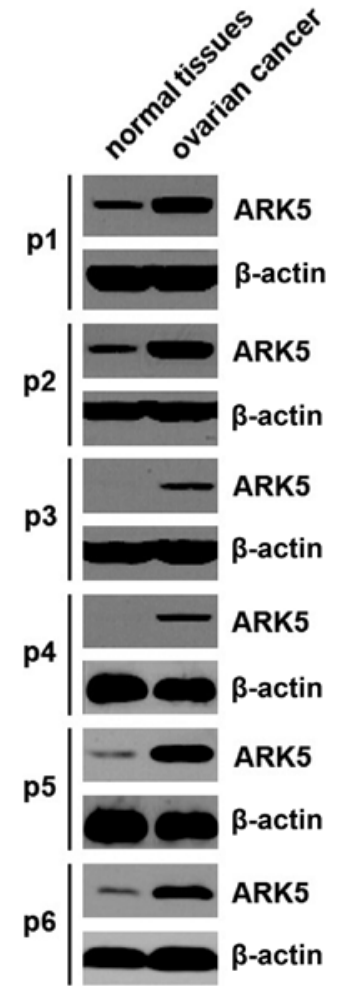

Figure 1. ARK5 is upregulated in ovarian cancer tissues. Western blotting for ARK5 in ovarian cancer tissues (C) and adjacent normal tissues (N). Patients were numbered no. 1-6. All 6 patients were diagnosed as ovarian cancer. $\beta$-actin was a loading control, $n=6$.

its expression was highest in HEY cells among the six ovarian cancer cell lines (Fig. 2A). To identify whether ARK5 was associated with EMT in ovarian cancer, we performed western blotting to detect epithelial and mesenchymal markers in EG cells (ARK5-negative) and HEY cells (ARK5-positive). The results showed that the expression of E-cadherin and $\alpha$-catenin were significantly elevated in EG cells (ARK5-negative cells), compared with HEY cells (ARK5-positive cells) (Fig. 2B). By contrast, the expression of $\mathrm{N}$-cadherin, vimentin and $\alpha$-SMA protein was upregulated in HEY cells (ARK5-positive cells) (Fig. 2B). Thus, ARK5 may be associated with EMT in ovarian cancer.

In order to assess the role of ARK5 in ovarian cancer, we transfected EG cells with ARK5-expressing plasmids, followed by western blotting. ARK5 protein was significantly increased in the cells transfected with ARK5-expressing plasmids (Fig. 2C) and its overexpression caused significant changes in EG cell morphology (EMT) (Fig. 2D). To verify that the changes in cell morphology were caused by EMT, the expression levels of epithelial and mesenchymal markers were compared in EG cells transfected with ARK5-expressing plasmids with EG cells transfected with empty vectors. The results revealed that the epithelial markers (E-cadherin) were consistently repressed, whereas the mesenchymal markers ( $\beta$-catenin, fibronectin, vimentin and $\alpha$-SMA) were induced by ARK5 overexpression in EG cells (Fig. 2E). EMT resulted in increased cell invasion and migration (29-31). Thus, ARK5 also affected invasion and migration in EG cells. To determine the reason for this result, we performed invasion, migration, and wound-healing assays. We found that ARK5 resulted in 
A

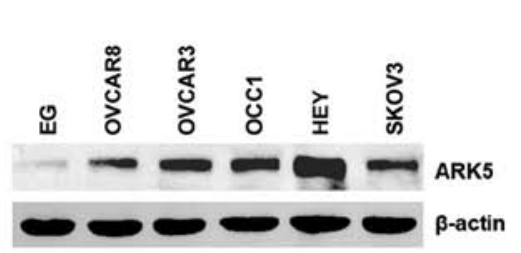

B

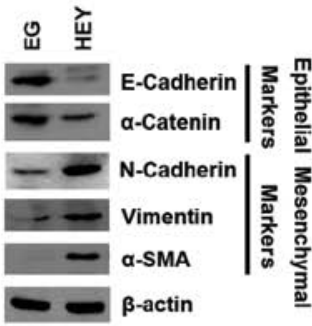

C
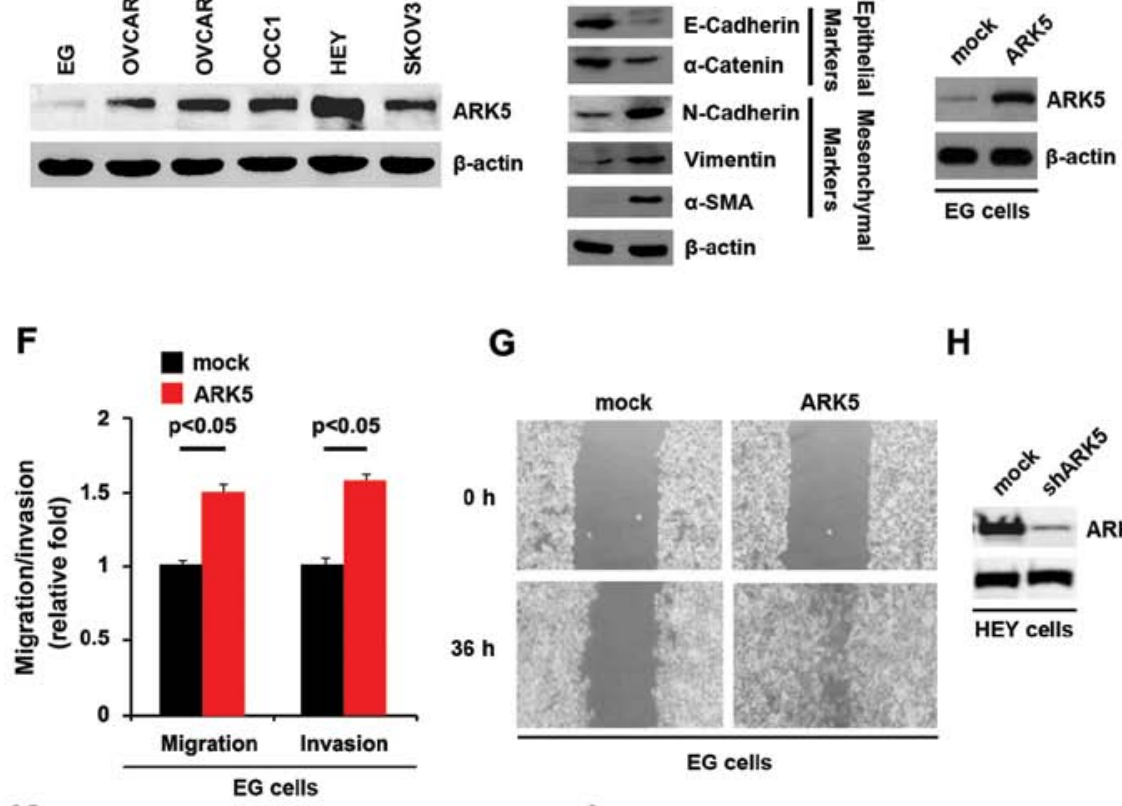

$\mathbf{K}$

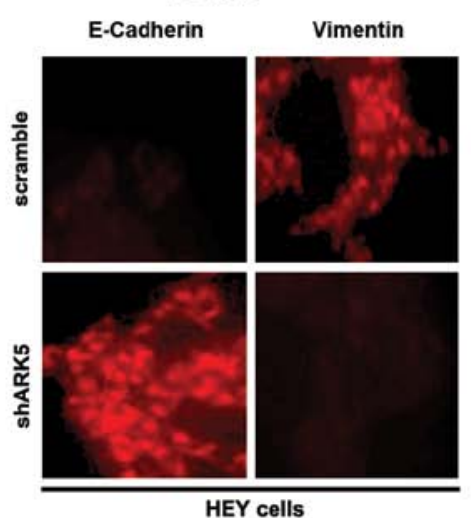

H

G

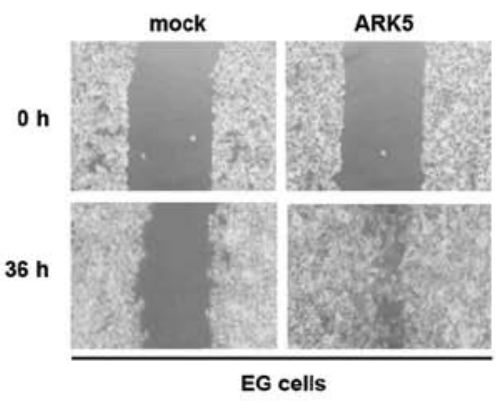

$\mathbf{L}$

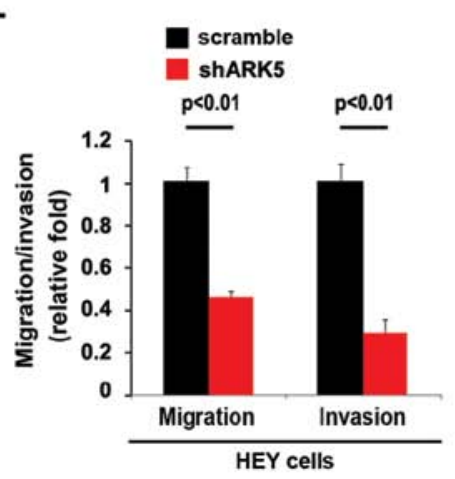

D

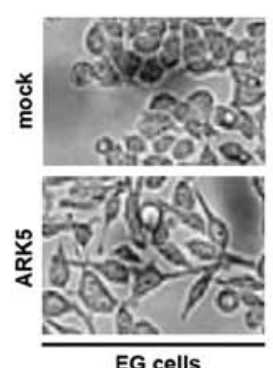

EG cells

I
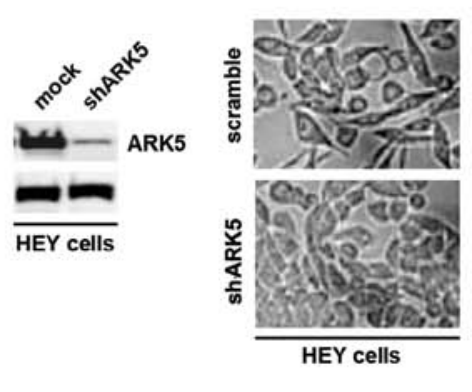

E

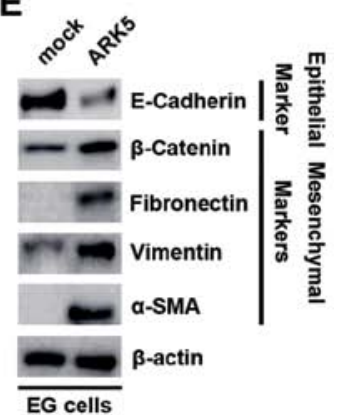

M

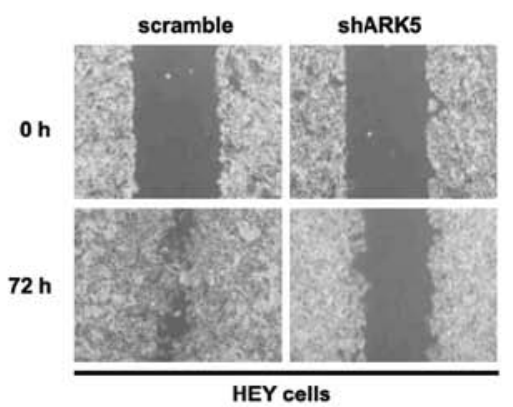

Figure 2. (A) ARK5 promotes EMT in ovarian cancer cells. Western blotting for ARK5 in EG, OVCAR8, OVCAR3, OCC1, HEY and SKOV3 ovarian cancer cell lines. $\beta$-actin served as a loading control, $n=3$. (B) Western blotting for E-cadherin, $\alpha$-catenin, $N$-cadherin, vimentin and $\alpha$-SMA in ovarian cancer EG and HEY cells. $\beta$-actin served as a loading control, $n=3$. (C) Western blotting for ARK5 in EG cells transfected with ARK5-expressing plasmids. Mock groups were transfected with empty vectors. $\beta$-actin served as a loading control, $n=3$. (D) EG cells were transfected as indicated. Cells were then photographed, $n=3$. (E) Western blotting for E-cadherin, $\beta$-catenin, fibronectin, vimentin and $\alpha$-SMA in ovarian cancer cells transfected with ARK5-expressing plasmids. Mock groups were transfected with empty vectors. $\beta$-actin served as a loading control, $\mathrm{n}=3$. (F) Invasion and migration assays for EG cells transfected as indicated, $\mathrm{n}=3$. (G) Wound-healing assays for EG cells transfected with ARK5-expressing plasmids and empty vector (mock). The cell layer was photographed, $\mathrm{n}=3$. (H) Western blotting for ARK5 in ovarian cancer HEY cells transfected as indicated. $\beta$-actin served as a loading control, $\mathrm{n}=3$. (I) HEY cells were transfected as indicated. Cells were then photographed, $\mathrm{n}=3$. (J) Western blotting for SNAI1, TWIST, TGFB1, ZEB1 and TGFB2 in HEY cells transfected as indicated. $\beta$-actin served as a loading control, $n=3$. (K) Immunocytochemistry for HEY cells transfected as indicated, $n=3$. (L) Invasion and migration assays for HEY cells transfected as indicated, $n=3$. (M) Wound-healing assays for HEY cells transfected as indicated. The cell layer was photographed, $n=3$. EMT, epithelial-mesenchymal transition.

enhanced invasion (Fig. 2F) and migration (Fig. 2F and G) in the cells.

As shown above ARK5 overexpression promoted EMT in EG cells. To provide further evidence that ARK5 was involved in EMT of ovarian cancer, we studied the effects of an inhibitor of ARK5, shARK5. After stable transfection, ARK5 expression was detected by western blotting. The results showed that shARK5 significantly downregulated ARK5 protein expression in HEY cells (Fig. 2H). After transfection, we observed that silencing ARK5 caused significant changes in HEY cell morphology (MET) (Fig. 2I). To confirm that silencing ARK5 was associated with MET in ovarian cancer, we performed western blotting to detect the expression of mesenchymal markers (SNAI1, TWIST, TGFB1, ZEB1 and TGFB2). The results demonstrated that the expression of SNAI1, TWIST, TGFB1 and ZEB1 was evidently attenuated by silencing ARK5 (Fig. 2J). We also performed immunocytochemistry to detect the expression of E-cadherin (epithelial markers) and vimentin (mesenchymal markers). The results showed that silencing ARK5 significantly upregulated E-cadherin expression and downregulated vimentin expression (Fig. 2K). Given that ARK5 overexpression promoted migration and invasion in EG cells (ARK5-negative), we hypothesized that silencing ARK5 impaired the ability of migration and invasion in 
A
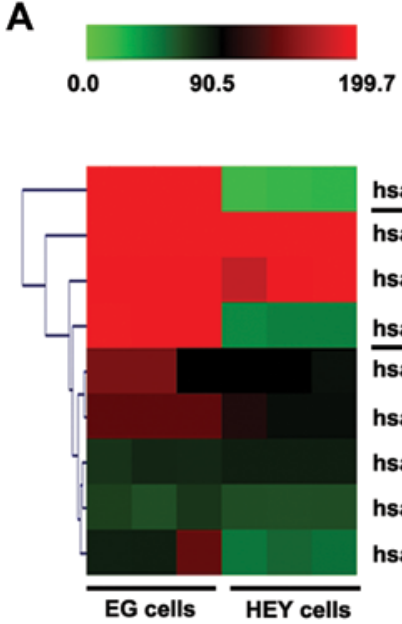

B

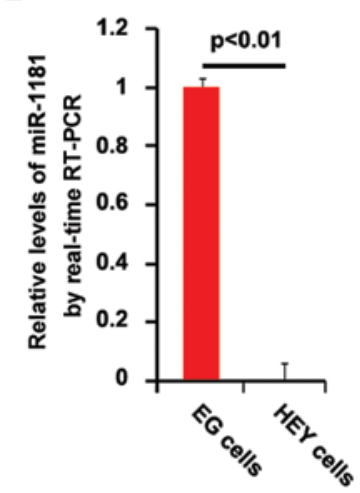

D
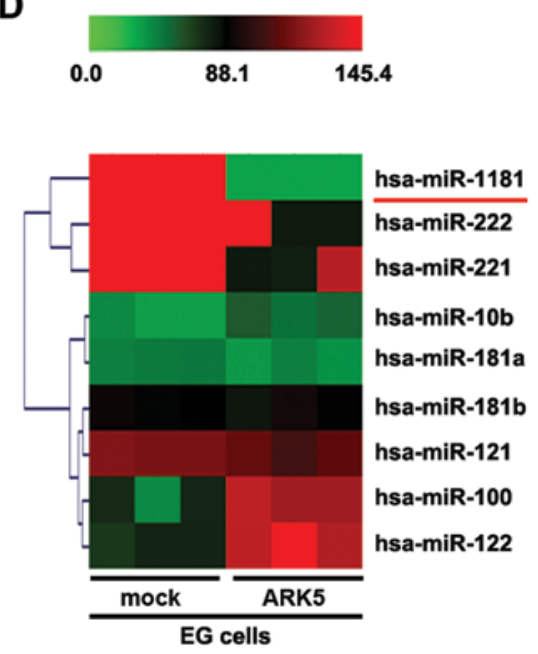

E
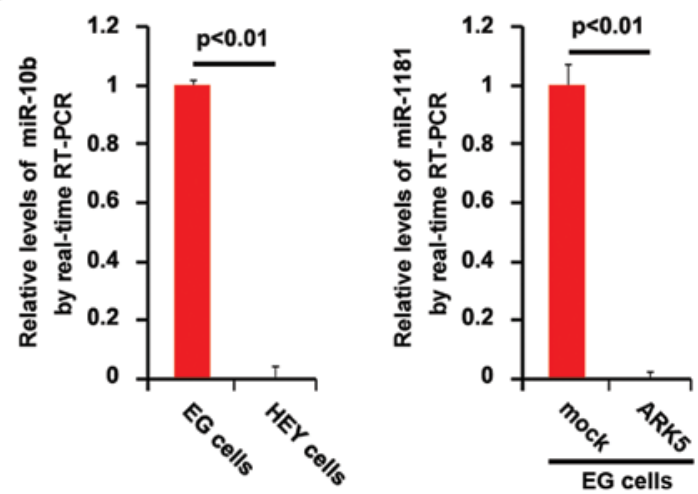

Figure 3. miR-1181 is suppressed by ARK5 in ovarian cancer. (A) Partial heat map of miRNA microarray analysis for EG and HEY cells, n=3. (B) RT-PCR for miR-1181 in EG and HEY cells. U6 served as a loading control, $n=3$. (C) RT-PCR for miR-10b in EG and HEY cells. U6 was a loading control, $n=3$. (D) Partial heat map of miRNA microarray analysis for EG cells transfected as indicated. Mock groups were transfected with empty vectors, $\mathrm{n}=3$. (E) RT-PCR for miR-1181 in EG cells transfected as indicated. Mock groups were transfected with empty vectors. U6 served as a loading control, $\mathrm{n}=3$.

HEY cells (ARK5-positive). Thus, we performed invasion, migration, and wound-healing assays to observe the effect of silencing ARK5 on invasion and migration. The results confirmed that silencing ARK5 inhibited invasion (Fig. 2L) and migration (Fig. 2L and M) in HEY cells. Thus, ARK5 promoted EMT in ovarian cancer cells.

ARK5 suppresses miR-1181 expression in ovarian cancer cells. miRNAs, the small non-coding RNA molecules that suppress gene expression by interacting with the 3'UTRs of target mRNAs, have also been associated with EMT and cancer (4,32-34). Moreover, since oncogenes exert their functions by regulating miRNA expression in tumor (35), we investigated whether ARK5 affected miRNA expression in ovarian cancer cells.

miRNA microarrays were performed. RNAs isolated from EG and HEY cells were hybridized to a custom miRNA microarray platform. After hybridization, quantification and normalization were carried out three times, a number of miRNAs, particularly miR-1181 and miR-10b, were downregulated >150-fold in HEY cells (ARK5-positive), compared with EG cells (ARK5-negative) (Fig. 3A). We also performed RT-PCR to confirm the results of miRNA microarray.Consistent with miRNA microarray, the results of RT-PCR showed that
miR-1181 and miR-10b were significantly downregulated in HEY cells, compared with EG cells (Fig. 3B and C). The results suggested that miR-1181 and miR-10b inhibition may be associated with the overexpression of ARK5 in ovarian cancer cells. To identify the association between ARK5 and the two miRNAs, we transfected EG cells (ARK5-negative) with ARK5-expressing plasmids or empty vectors and then miRNA microarrays were performed. We found that ARK5 downregulated miR-1181 expression $>100$-fold, although it did not affect miR-10b expression in EG cells (Fig. 3D). Moreover, the results of RT-PCR confirmed that ARK5 significantly inhibited miR-1181 expression in EG cells (Fig. 3E).

miR-1181 promotes MET in ovarian cancer cells. Given that ARK5 promoted EMT in ovarian cancer cells and inhibited miR-1181 expression in the cells, we examined whether miR-1181 inhibited EMT and promoted MET, while ARK5 promoted EMT by inhibiting miR-1181 expression in ovarian cancer cells. To identify the role of miR-1181 in regulating EMT in ovarian cancer cells, HEY cells were transfected with pre-miR-1181 and control miR. Following transfection, miR-1181 expression was detected by RT-PCR and the results showed that miR-1181 was increased by pre-miR-1181 in HEY cells (Fig. 4A). Additionally, its overexpression caused 
A

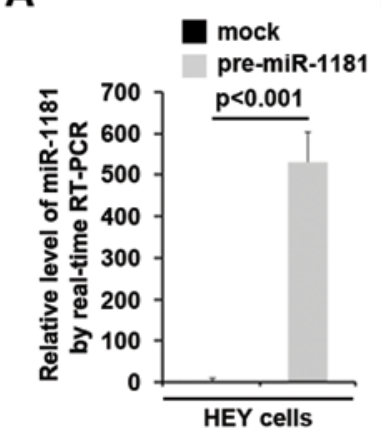

B

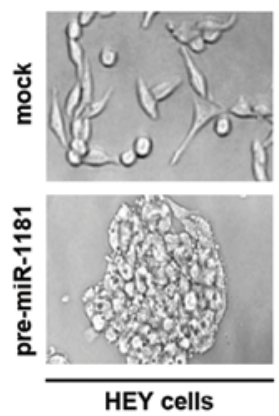

C

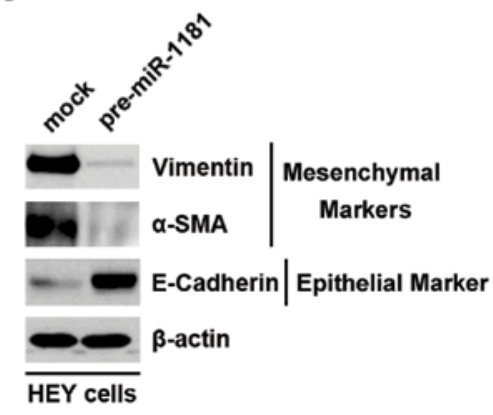

D

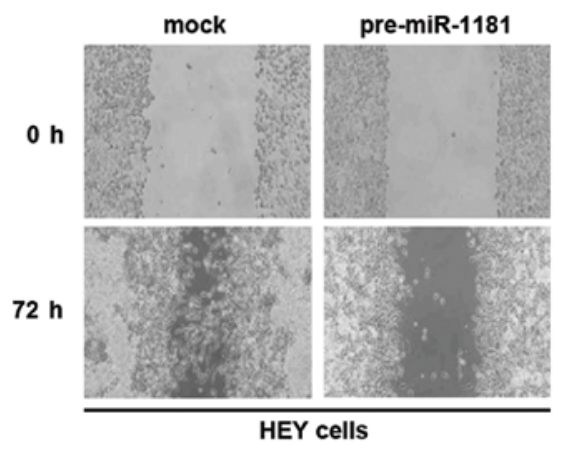

$\mathbf{F}$

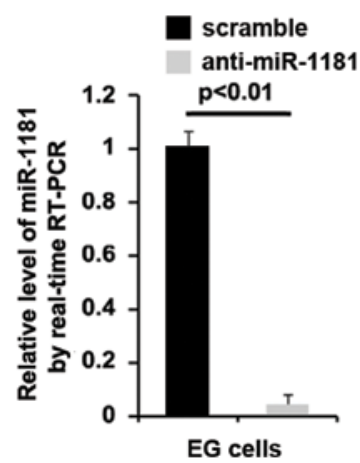

E

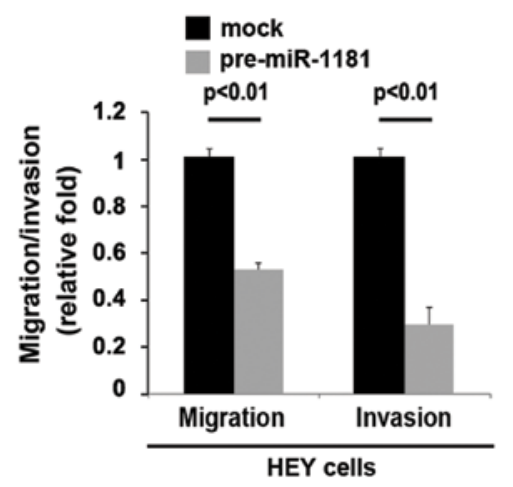

H

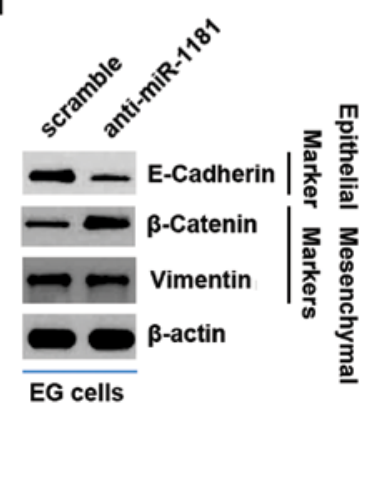

Figure 4. miR-1181 promotes MET in ovarian cancer. (A) RT-PCR for miR-1181 in HEY cells transfected as indicated. Mock groups were transfected with control miR. U6 served as a loading control, $n=3$. (B) HEY cells were transfected as indicated. Mock groups were transfected with control miR. Cells were then photographed, $n=3$. (C) Western blotting for vimentin, $\alpha$-SMA and E-cadherin in HEY cells transfected with pre-miR-1181. Mock groups were transfected with control miR. $\beta$-actin served as a loading control, $n=3$. (D) Wound-healing assays for HEY cells transfected as indicated. Mock groups were transfected with control miR. The cell layer was photographed, $n=3$. (E) Invasion and migration assays for HEY cells transfected as indicated. Mock groups were transfected with control miR, $n=3$. (F) RT-PCR for miR-1181 in EG cells transfected as indicated. U6 served as a loading control, n=3. (G) EG cells were transfected as indicated. Cells were then photographed, $n=3$. $(\mathrm{H})$ Western blotting for E-cadherin, $\beta$-catenin and vimentin in EG cells transfected as indicated. $\beta$-actin served as a loading control, $n=3$. MET, mesenchymal-epithelial transition.

significant changes in HEY cell morphology (MET) (Fig. 4B). To identify whether miR-1181 promoted MET, we performed western blotting to detect mesenchymal and epithelial markers. The results demonstrated that the expression of vimentin and $\alpha$-SMA were significantly suppressed in HEY cells transfected with pre-miR-1181, compared with the cells transfected with control miR (Fig. 4C). We also identified that E-cadherin was upregulated by pre-miR-1181 in HEY cells (Fig. 4C). Wound-healing, migration and invasion assays were performed to detect the migration and invasion of HEY cells transfected with pre-miR-1181 or control miR. Ectopic miR-1181 significantly inhibited motility (Fig. 4D and E) and invasion (Fig. 4E).

As shown above miR-1181 promoted MET in ovarian cancer cells. Thus, to provide further evidence that the roles of miR-1181 were involved in MET of ovarian cancer cells, we studied the effects of an inhibitor of miR-1181 and anti-miR-1181. After stable transfection, miR-1181 expression was detected by RT-PCR in EG cells. The results showed that anti-miR-1181 significantly downregulated miR-1181 expression in EG cells (Fig. 4F). We also observed the morphology of EG cells transfected with anti-miR-1181, compared with the cells transfected with scramble. The results showed that contrary to miR-1181, silencing miR-1181 significantly changed EMT (Fig. 4G). Western blotting was performed to detect whether epithelial and mesenchymal markers were affected by anti-miR-1181 in EG ovarian cancer cells. The results showed that E-cadherin was significantly inhibited in the cells transfected with anti-miR-1181, whereas $\beta$-catenin was promoted by the transfection (Fig. $4 \mathrm{H}$ ). 
A

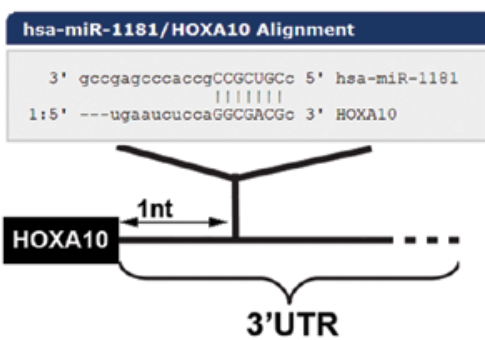

B

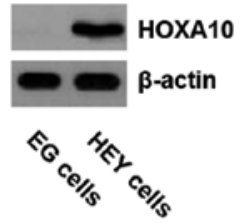

C

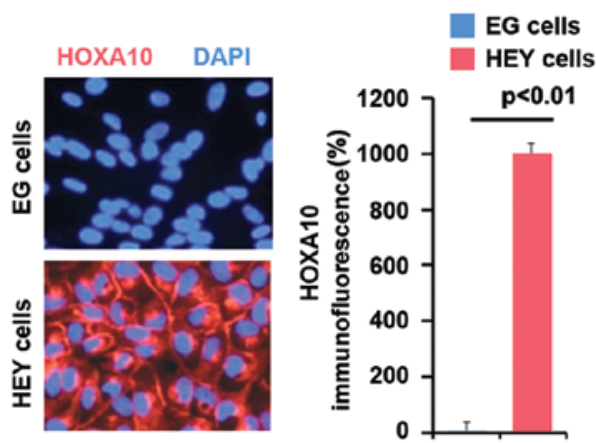

D

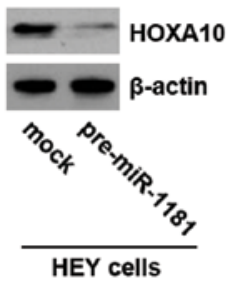

G
E

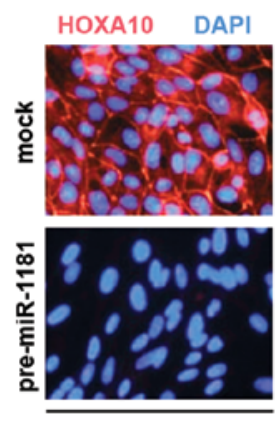

HEY cells

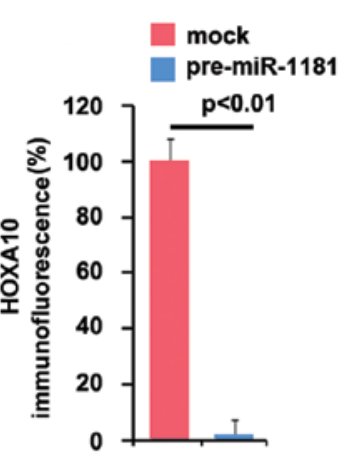

H
F

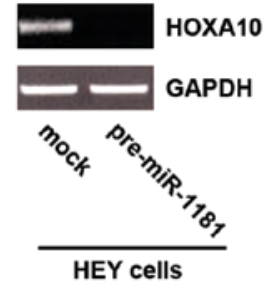

I

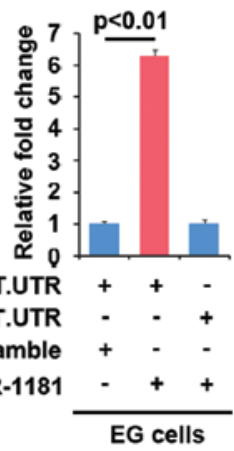

Figure 5. miR-1181 degrades HOXA10 by targeting its 3'UTR in ovarian cancer cells. (A) Vin Diagram showing the predicted miR-1181 targeting 3'UTR of HOXA10 mRNA from the database of miRanda. (B) Western blotting for HOXA10 protein in EG and HEY cells. $\beta$-actin was a loading control, $\mathrm{n}=3$. (C) Immunofluorescence analyses for EG and HEY cells. Top panel shows microscopic images of immunofluorescence staining of one representative experiment (magnification, x100). Bottom panel shows graphic presentation of mean fluorescence intensities, $n=3$. (D) Western blotting for HOXA10 protein in HEY cells infected as indicated. Mock groups were transfected with control miR. $\beta$-actin served as a loading control, $n=3$. (E) Immunofluorescence analyses for HEY cells transfected with pre-miR-1181 or control miR (mock). Left panel shows microscopic images of immunofluorescence staining of one representative experiment magnification, $x 100$ ). Top panel shows graphic presentation of mean fluorescence intensities, $n=3$. (F) RT-PCR for HOXA10 mRNA in HEY cells infected as indicated. GAPDH served as a loading control, $n=3$. (G) Diagram of HOXA10-3'UTR-containing reporter constructs. MUT, contains 4-basemutation at the miR-1181-target region, abolishing its binding. (H) Reporter assay for HEY cells, with co-transfection of 500 ng WT-or MUT-reporter and $50 \mathrm{nM}$ control-miR (mock) or pre-miR-1181 as indicated, $\mathrm{n}=3$. (I) Reporter assay for EG cells, with co-transfection of $500 \mathrm{ng}$ WT-or MUT-reporter and $50 \mathrm{nM}$ scramble or anti-miR-1181 as indicated, $n=3$.

miR-1181 degrades HOXA10 by targeting its 3'UTR in ovarian cancer. miRNAs are a new class of small ( $\sim 22$ nucleotide) non-coding RNAs that negatively regulate protein-coding gene expression by targeting mRNA degradation or translation inhibition (19-21). Thus, a search for downstream targets of miR-1181 in silico was conducted. The commonly used prediction algorithm-miRanda (http://www.microrna.org/microrna/ home.do) was utilized to analyze the predicted target genes of miR-1181. A number of target genes was identified, including HOXA10, which was overexpressed and promoted migration, and invasion in human ovarian cancer and correlated with poor survival (36). A schematic of predicted miR-1181-binding sites in the 3'UTR of HOXA10 mRNA is shown in Fig. 5A. miR-1181 was found to promote MET in ovarian cancer cells by targeting HOXA10 in ovarian cancer.

Given that miR-1181 expression was significantly upregulated in EG cells compared with HEY cells, we performed western blotting to detect HOXA10 protein in the two cell lines. The results showed that HOXA10 was detected in HEY cells, but not in EG cells (Fig. 5B). We also performed 


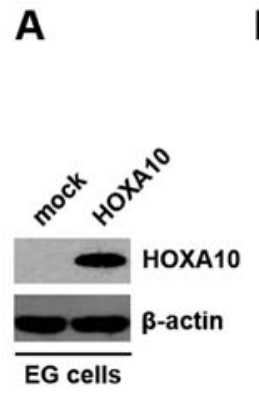

B

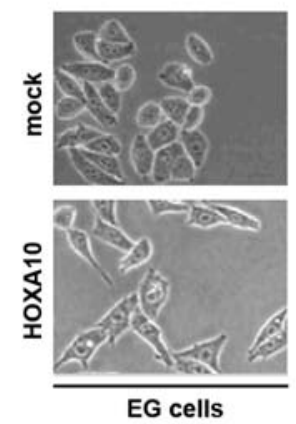

D

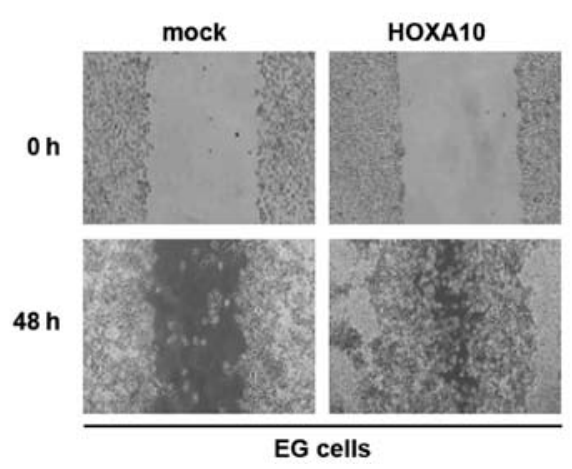

C

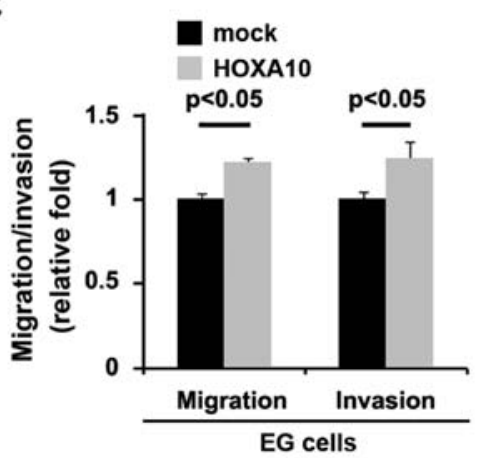

E

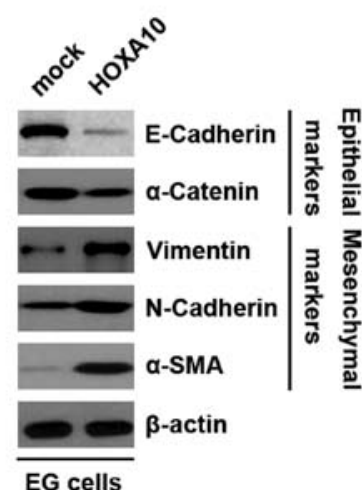

Figure 6. HOXA10 promotes EMT in ovarian cancer. (A) Western blotting for HOXA10 in EG cells transfected with HOXA10-expressing plasmids. Mock groups were transfected with empty vector. $\beta$-actin served as a loading control, $n=3$. (B) EG cells were transfected as indicated. Mock groups were transfected with empty vectors. Cells were then photographed, $n=3$. (C) Invasion and migration assays for EG cells transfected as indicated. Mock groups were transfected with empty vectors, $n=3$. (D) Wound-healing assays for EG cells transfected as indicated. Mock groups were transfected with empty vectors. The cell layer was photographed, $n=3$. (E) Western blotting for E-cadherin, $\alpha$-catenin, vimentin, $N$-cadherin and $\alpha$-SMA in EG cells transfected with HOXA10-expressing plasmids. Mock groups were transfected with empty vectors. $\beta$-actin served as a loading control, $n=3$. EMT, epithelial-mesenchymal transition.

immunofluorescence analyses in EG and HEY cells. The results showed that HOXA10 protein was evidently suppressed in the EG cells (Fig. 5C). The results suggested that HOXA10 was negatively associated with miR-1181 expression.

To confirm this finding, we performed western blotting in HEY cells transfected with pre-miR-1181 or control miR. The results showed that HOXA10 protein was evidently suppressed in the cells transfected with pre-miR-1181 (Fig. 5D). We also performed immunofluorescence analyses in the cells. Consistent with the results of western blotting, immunofluorescence analyses demonstrated that HOXA10 was reduced in HEY cells transfected with pre-miR-1181, compared with the control miR-transfected groups (Fig. 5E). We then performed RT-PCR to detect HOXA10 mRNA expression in HEY cells transfected with pre-miR-1181 or control miR. The results showed that HOXA10 mRNA (Fig. 5F) was significantly suppressed in the cells transfected with pre-miR-1181. To demonstrate the direct regulation of HOXA10 by miR-1181 through its 3'UTR, we constructed luciferase reporters with the targeting sequences of wild-type (HOXA10-WT-luc) and mutated HOXA10 3'UTRs (HOXA10-MUT-luc) (Fig. 5G). Both the wild-type and mutant reporters were introduced into HEY cells. The luciferase reporter assay showed that the luciferase activities of HOXA10-WT-luc plasmids were significantly suppressed in the cells transfected with pre-miR-1181, suggesting that miR-1181 targeted 3'UTR of HOXA10 mRNA (Fig. 5H). To determine whether miR-1181 targeted 3'UTR of HOXA10 at the predicted sites, we mutated four bases in the predicted sites (Fig. 5G). Mutant reporters were subsequently introduced into HEY cells as expected. The luciferase activities of HOXA10-MUT-luc were not suppressed by miR-1181 in HEY cells (Fig. 5H).

Given that miR-1181 overexpression inhibited HOXA10WT-luc plasmids at the predicted sites, we investigated whether silencing miR-1181 affected activity of the HOXA10-WT-luc plasmids. Thus, the luciferase reporter assay was performed again and the results showed that contrary to pre-miR-1181, anti-miR-1181 significantly promoted the luciferase activity of HOXA10-WT-luc in EG cells (Fig. 5I). Moreover, mutant reporters were introduced into EG cells, although the luciferase activities of HOXA10-MUT-luc were not affected by anti-miR-1181 in EG cells (Fig. 5I). The results suggested that miR-1181 degraded HOXA10 by targeting the specific sites predicted in silico in ovarian cancer cells.

HOXA10 promotes EMT in ovarian cancer cells. As shown above, miR-1181 degrades HOXA10 by targeting its 3'UTR in ovarian cancer cells. Thus, we examined the roles of HOXA10 in EMT of ovarian cancer.

To identify the role of HOXA10 in regulating EMT in ovarian cancer, the cells were transfected with HOXA10expressing plasmids. After stable transfection, HOXA10 protein expression was detected by western blotting and the results showed that HOXA10 protein was increased by HOXA10-expressing plasmids in the cells (Fig. 6A). HOXA10 overexpression caused significant changes in EMT (Fig. 6B). 
In addition, to identify whether HOXA10 affected invasion and migration, we performed invasion, migration, and wound-healing assays. HOXA10 resulted in increased cell invasion (Fig. 6C) and migration (Fig. 6C and D) in EG cells.

To verify that the changes in cell morphology and characteristics of invasion and migration were caused by EMT, the expression levels of epithelial and mesenchymal markers were compared in EG cells transfected with HOXA10-expressing plasmids or empty vectors. The results revealed that the epithelial markers (E-cadherin and $\alpha$-catenin) were consistently repressed, whereas mesenchymal markers (vimentin, $\mathrm{N}$-cadherin and $\alpha$-SMA) were induced by HOXA10 overexpression in EG cells (Fig. 6E).

\section{Discussion}

EMT is a crucial developmental program in which immotile epithelial cells acquire mesenchymal features. Activation of EMT triggers tumor cell invasion and dissemination and is thus considered as the initiating step of cancer metastasis $(37,38)$. Epithelial cells are characterized by several features including, cohesive interactions among cells, facilitating the formation of continuous cell layers; existence of three membrane domains: apical, lateral and basal; presence of tight junctions between apical and lateral domains; apicobasal polarized distribution of the various organelles and cytoskeleton components; expression of epithelial markers such as E-cadherin and $\alpha$-catenin; lack of mobility of individual epithelial cells with respect to their local environment (39-41). Mesenchymal architectures are different from epithelial ones including features such as loose or no interactions among cells; no clear apical and lateral membranes; no apico-basal polarized distribution of organelles and cytoskeleton components; and expression of mesenchymal markers, such as $\mathrm{N}$-cadherin, vimentin, $\beta$-catenin, $\alpha$-SMA, SNAI1, TWIST, TGFB1, ZEB1 and TGFB2; motile cells having invasive properties $(39,42-44)$. During EMT, cell-cell junctions are altered, cells lose epithelial polarity, express the mesenchymal markers and the resulting reorganization of the actin cytoskeleton supports cell migration. We found that ARK5 protein expression was positively associated with mesenchymal markers in ovarian cancer cell lines. Moreover, its overexpression caused significant changes in EG cell morphology (EMT) and unregulated mesenchymal markers and downregulated epithelial markers. Contrary to its overexpression, silencing ARK5 caused significant changes in HEY cell morphology (MET) and suppressed expression of mesenchymal markers, such as vimentin, SNAI1, TWIST, TGFB1, ZEB1 and vimentin as well as upregulated epithelial marker E-cadherin.

miRNAs, the small non-coding RNA molecules that suppress gene expression by interacting with the 3 ' untranslated regions (3'UTRs) of target mRNAs, have also been associated with EMT and cancer (4,32-34). Epithelial-mesenchymal transition (EMT) has recently been associated with stem-cell phenotype $(27,45)$. It has been reported that miR-1181 can inhibit stem cell-like phenotypes and suppresses SOX2 and STAT3 in human pancreatic cancer (26). Consistent with previous studies, we confirmed that miR-1181 promoted MET in ovarian cancer cells and its expression was significantly downregulated by ARK5. However, whether ARK5 and miR-1181 are linked to the stem-cell phenotype of ovarian cancer remains to be determined.

An increased expression of HOXA10 was detected in almost all ovarian carcinomas (46). In the present study, we confirmed that HOXA10 promoted EMT in ovarian cancer. Elucidating the mechanism that miR-1181 promotes MET in ovarian cancer by downregulating HOXA10 may be useful to understand the mechanism of EMT. Thus, targeting ARK5 and restoration of miR-1181 may be a promising therapeutic approach to suppress HOXA10-mediated EMT. However, the role of the ARK5/miR-1181/HOXA10 axis remains to be confirmed in vivo.

\section{References}

1. Nieto MA: The ins and outs of the epithelial to mesenchymal transition in health and disease. Annu Rev Cell Dev Biol 27: 347-376, 2011.

2. Savagner P, Yamada KM and Thiery JP: The zinc-finger protein slug causes desmosome dissociation, an initial and necessary step for growth factor-induced epithelial-mesenchymal transition. J Cell Biol 137: 1403-1419, 1997.

3. Thiery JP: Epithelial-mesenchymal transitions in tumour progression. Nat Rev Cancer 2: 442-454, 2002.

4. Chang CJ, Chao CH, Xia W, Yang JY, Xiong Y, Li CW, Yu WH, Rehman SK, Hsu JL, Lee HH, et al: p53 regulates epithelialmesenchymal transition and stem cell properties through modulating miRNAs. Nat Cell Biol 13: 317-323, 2011.

5. Gravdal K, Halvorsen OJ, Haukaas SA and Akslen LA: A switch from E-cadherin to $\mathrm{N}$-cadherin expression indicates epithelial to mesenchymal transition and is of strong and independent importance for the progress of prostate cancer. Clin Cancer Res 13: 7003-7011, 2007.

6. Hader C, Marlier A and Cantley L: Mesenchymal-epithelial transition in epithelial response to injury: The role of Foxc2. Oncogene 29: 1031-1040, 2010.

7. Suzuki A, Kusakai G, Kishimoto A, Lu J, Ogura T, Lavin MF and Esumi H: Identification of a novel protein kinase mediating Akt survival signaling to the ATM protein. J Biol Chem 278: 48-53, 2003.

8. Suzuki A, Kusakai G, Kishimoto A, Lu J, Ogura T and Esumi H: ARK5 suppresses the cell death induced by nutrient starvation and death receptors via inhibition of caspase 8 activation, but not by chemotherapeutic agents or UV irradiation. Oncogene 22: 6177-6182, 2003.

9. Suzuki A, Kusakai G, Kishimoto A, Shimojo Y, Miyamoto S, Ogura T, Ochiai A and Esumi H: Regulation of caspase- 6 and FLIP by the AMPK family member ARK5. Oncogene 23: 7067-7075, 2004.

10. Kusakai G, Suzuki A, Ogura T, Kaminishi M and Esumi H: Strong association of ARK5 with tumor invasion and metastasis. J Exp Clin Cancer Res 23: 263-268, 2004.

11. Kusakai G, Suzuki A, Ogura T, Miyamoto S, Ochiai A, Kaminishi M and Esumi H: ARK5 expression in colorectal cancer and its implications for tumor progression. Am J Pathol 164: 987-995, 2004.

12. Suzuki A, Lu J, Kusakai G, Kishimoto A, Ogura T and Esumi H: ARK5 is a tumor invasion-associated factor downstream of Akt signaling. Mol Cell Biol 24: 3526-3535, 2004.

13. Chen P, Li K, Liang Y, Li L and Zhu X: High NUAK1 expression correlates with poor prognosis and involved in NSCLC cells migration and invasion. Exp Lung Res 39: 9-17, 2013.

14. Shi L, Zhang B, Sun X, Lu S, Liu Z, Liu Y, Li H, Wang L, Wang X and Zhao C: MiR-204 inhibits human NSCLC metastasis through suppression of NUAK1. Br J Cancer 111: 2316-2327, 2014.

15. Bell RE, Khaled M, Netanely D, Schubert S, Golan T, Buxbaum A, Janas MM, Postolsky B, Goldberg MS, Shamir R, et al: Transcription factor/microRNA axis blocks melanoma invasion program by miR-211 targeting NUAK1. J Invest Dermatol 134: 441-451, 2014

16. Lu S, Niu N, Guo H, Tang J, Guo W, Liu Z, Shi L, Sun T, Zhou F, $\mathrm{Li} \mathrm{H}$, et al: ARK5 promotes glioma cell invasion, and its elevated expression is correlated with poor clinical outcome. Eur J Cancer 49: 752-763, 2013. 
17. Chang XZ, Yu J, Liu HY, Dong RH and Cao XC: ARK5 is associated with the invasive and metastatic potential of human breast cancer cells. J Cancer Res Clin Oncol 138: 247-254, 2012.

18. Cui J, Yu Y, Lu GF, Liu C, Liu X, Xu YX and Zheng PY: Overexpression of ARK5 is associated with poor prognosis in hepatocellular carcinoma. Tumour Biol 34: 1913-1918, 2013.

19. Lee RC, Feinbaum RL and Ambros V: The C. elegans heterochronic gene lin-4 encodes small RNAs with antisense complementarity to lin-14. Cell 75: 843-854, 1993.

20. Pasquinelli AE, Reinhart BJ, Slack F, Martindale MQ, Kuroda MI, Maller B, Hayward DC, Ball EE, Degnan B, Müller P, et al: Conservation of the sequence and temporal expression of let-7 heterochronic regulatory RNA. Nature 408: 86-89, 2000.

21. Reinhart BJ, Slack FJ, Basson M, Pasquinelli AE, Bettinger JC, Rougvie AE, Horvitz HR and Ruvkun G: The 21-nucleotide let-7 RNA regulates developmental timing in Caenorhabditis elegans. Nature 403: 901-906, 2000.

22. Esteller M: Non-coding RNAs in human disease. Nat Rev Genet 12: 861-874, 2011.

23. Esquela-Kerscher A and Slack FJ: Oncomirs-microRNAs with a role in cancer. Nat Rev Cancer 6: 259-269, 2006.

24. Garzon R, Calin GA and Croce CM: MicroRNAs in cancer. Annu Rev Med 60: 167-179, 2009.

25. Slack FJ and Weidhaas JB: MicroRNA in cancer prognosis. N Engl J Med 359: 2720-2722, 2008

26. Jiang J, Li Z, Yu C, Chen M, Tian S and Sun C: MiR-1181 inhibits stem cell-like phenotypes and suppresses SOX2 and STAT3 in human pancreatic cancer. Cancer Lett 356: 962-970, 2015.

27. Polyak K and Weinberg RA: Transitions between epithelial and mesenchymal states: Acquisition of malignant and stem cell traits. Nat Rev Cancer 9: 265-273, 2009.

28. Liao XH, Lu DL, Wang N, Liu LY, Wang Y, Li YQ, Yan TB Sun $\mathrm{XG}, \mathrm{Hu} \mathrm{P}$ and Zhang TC: Estrogen receptor $\alpha$ mediates proliferation of breast cancer MCF-7 cells via a p21/PCNA/E2F1dependent pathway. FEBS J 281: 927-942, 2014.

29. Zuo JH, Zhu W, Li MY, Li XH, Yi H, Zeng GQ, Wan XX, He QY, $\mathrm{Li} \mathrm{JH}, \mathrm{Qu} \mathrm{JQ}$, et al: Activation of EGFR promotes squamous carcinoma SCC10A cell migration and invasion via inducing EMT-like phenotype change and MMP-9-mediated degradation of E-cadherin. J Cell Biochem 112: 2508-2517, 2011.

30. Jung H, Lee KP, Park SJ, Park JH, Jang YS, Choi SY, Jung JG, Jo K, Park DY, Yoon JH, et al: TMPRSS4 promotes invasion, migration and metastasis of human tumor cells by facilitating an epithelial-mesenchymal transition. Oncogene 27: 2635-2647, 2008.

31. Christiansen JJ and Rajasekaran AK: Reassessing epithelial to mesenchymal transition as a prerequisite for carcinoma invasion and metastasis. Cancer Res 66: 8319-8326, 2006.

32. Bracken CP, Gregory PA, Kolesnikoff N, Bert AG, Wang J, Shannon MF and Goodall GJ: A double-negative feedback loop between ZEB1-SIP1 and the microRNA-200 family regulates epithelial-mesenchymal transition. Cancer Res 68: 7846-7854, 2008
33. Gregory PA, Bracken CP, Bert AG and Goodall GJ: MicroRNAs as regulators of epithelial-mesenchymal transition. Cell Cycle 7: 3112-3118, 2008

34. Korpal M,Lee ES, Hu G and Kang Y: The miR-200 family inhibits epithelial-mesenchymal transition and cancer cell migration by direct targeting of E-cadherin transcriptional repressors $Z E B 1$ and ZEB2. J Biol Chem 283: 14910-14914, 2008.

35. Ma L, Young J, Prabhala H, Pan E, Mestdagh P, Muth D, TeruyaFeldstein J, Reinhardt F, Onder TT, Valastyan S, et al: miR-9, a MYC/MYCN-activated microRNA, regulates E-cadherin and cancer metastasis. Nat Cell Biol 12: 247-256, 2010.

36. Li B, Jin H, Yu Y, Gu C, Zhou X, Zhao N and Feng Y: HOXA10 is overexpressed in human ovarian clear cell adenocarcinoma and correlates with poor survival. Int J Gynecol Cancer 19: $1347-1352,2009$

37. Tsuji T, Ibaragi S and Hu GF: Epithelial-mesenchymal transition and cell cooperativity in metastasis. Cancer Res 69: 7135-7139, 2009.

38. Gavert $\mathrm{N}$ and Ben-Ze'ev A: Epithelial-mesenchymal transition and the invasive potential of tumors. Trends Mol Med 14: 199-209, 2008.

39. Larue L and Bellacosa A: Epithelial-mesenchymal transition in development and cancer: Role of phosphatidylinositol 3' kinase/ AKT pathways. Oncogene 24: 7443-7454, 2005.

40. Vasioukhin V, Bauer C, Degenstein L, Wise B and Fuchs E: Hyperproliferation and defects in epithelial polarity upon conditional ablation of alpha-catenin in skin. Cell 104: 605-617, 2001.

41. Schlegelmilch K, Mohseni M, Kirak O, Pruszak J, Rodriguez JR, Zhou D, Kreger BT, Vasioukhin V, Avruch J, Brummelkamp TR, et al: Yap1 acts downstream of $\alpha$-catenin to control epidermal proliferation. Cell 144: 782-795, 2011.

42. Masszi A, Di Ciano C, Sirokmány G, Arthur WT, Rotstein OD, Wang J, McCulloch CA, Rosivall L, Mucsi I and Kapus A: Central role for Rho in TGF-beta1-induced alpha-smooth muscle actin expression during epithelial-mesenchymal transition. Am J Physiol Renal Physiol 284: F911-F924, 2003.

43. Cano A, Pérez-Moreno MA, Rodrigo I, Locascio A, Blanco MJ, del Barrio MG, Portillo F and Nieto MA: The transcription factor snail controls epithelial-mesenchymal transitions by repressing E-cadherin expression. Nat Cell Biol 2: 76-83, 2000

44. Kang Y and Massagué J: Epithelial-mesenchymal transitions: Twist in development and metastasis. Cell 118: 277-279, 2004.

45. Mani SA, Guo W, Liao MJ, Eaton EN, Ayyanan A, Zhou AY, Brooks M, Reinhard F, Zhang CC, Shipitsin M, et al: The epithelial-mesenchymal transition generates cells with properties of stem cells. Cell 133: 704-715, 2008.

46. Cheng W, Jiang Y, Liu C, Shen O, Tang W and Wang X: Identification of aberrant promoter hypomethylation of HOXA10 in ovarian cancer. J Cancer Res Clin Oncol 136: 1221-1227, 2010 . 